\title{
Downregulation of MicroRNA-4463 Attenuates High-Glucose- and Hypoxia- Induced Endothelial Cell Injury by Targeting PNUTS
}

\author{
Xuemei Hea,d Chao Du ${ }^{\mathrm{b}}$ Yan Zou ${ }^{\mathrm{b}}$ Yang Long ${ }^{\mathrm{a}}$ Can Huang ${ }^{\mathrm{c}}$ Feng Chen ${ }^{\mathrm{a}}$ \\ Yanzheng $\mathrm{He}^{c}$ Xiangyu Zhou ${ }^{\mathrm{b}}$ \\ aExperimental Medicine Center, the Affiliated Hospital of Southwest Medical University, Luzhou, \\ bDepartment of Thyroid and Vascular Surgery, the Affiliated Hospital of Southwest Medical University, \\ Luzhou, 'Southwest Medical University, Luzhou, 'Collaborative Innovation Center for Prevention and \\ Treatment of Cardiovascular Disease of Sichuan Province, Southwest Medical University, Luzhou, China
}

\section{Key Words}

miR-4463 • Macrovascular complications • Apoptosis • AKT

\begin{abstract}
Background/Aims: Vascular complications are the main reasons for disability and mortality associated with type 2 diabetes mellitus (T2DM) and numerous microRNAs (miRNAs) are involved in this process. Our previous study demonstrated that miR-4463 was increased in the plasma of T2DM patients combined with arteriosclerosis of low extremity artery (ASO). However, the role of miR-4463 remains unclear. Methods: miR-4463 expression in the vascular tissues of patients with ASO and T2DM and in human umbilical vein endothelial cells (HUVECs) was detected by qPCR. Cell survival and apoptosis was analyzed via Cell Counting Kit- 8 and flow cytometry assays, respectively. Protein expression was determined by Western blot and protein subcellular localization was detected with immunofluorescence. A dualluciferase assay was used to elucidate the target gene of miR-4463. Results: miR-4463 was elevated in the vascular tissues of patients with T2DM and ASO. In HUVECs, both $25 \mathrm{mmol} / \mathrm{L}$ glucose (high glucose, HG) and hypoxia induced miR-4463 expression. Downregulation of miR-4463 promoted HUVEC survival and reduced cell apoptosis under HG and/or hypoxic conditions by facilitating the expression of protein phosphatase- 1 nuclear targeting subunit (PNUTS), X-linked inhibitor of apoptosis protein (XIAP), p-AKT, p-Bad, increased the Bcl-2/Bax ratio, as well as downregulated cleaved caspase 3 expression. Mechanistically, we identified PNUTS as a direct target gene of miR-4463. Both the inhibition of AKT phosphorylation and silencing of PNUTS diminished the effect of miR-4463 on HUVEC apoptosis. Moreover, downregulation of miR-4463 enhanced PNUTS to enable PTEN nuclear localization, which
\end{abstract}


resulted in AKT phosphorylation. Conclusion: Our results suggest that downregulation of miR-4463 attenuates cell apoptosis by directly enhancing PNUTS expression to promote PTEN nuclear localization, subsequently activating AKT signaling pathway in HUVECs under HG and/ or hypoxic conditions.

(C) 2018 The Author(s)

Published by S. Karger AG, Basel

\section{Introduction}

Diabetes mellitus (DM) characterized by hyperglycemia is probably the most common metabolic disease worldwide and has become a global threat to human health [1,2]. Among life-threatening DM cases, the majority are type 2 DM (T2DM), and associated complications include long-term damage, dysfunction, and failure of vital organs such as eyes, kidneys, nerves, and blood vessels. In particular, macrovascular complications have become the primary cause of disability and death $[3,4]$.

Vascular endothelial cell (EC) death and dysfunction are associated with a reduced thromboresistance and vasodilation of the vessel wall and may be an important risk factor of vascular complications in DM. Hyperglycemia-induced EC dysfunction and damage are the early steps in this process. High-glucose (HG) treatment readily induces ECs to be apoptotic, pro-inflammatory or anti-angiogenic. MicroRNAs (miRNAs) are endogenous 18- to 25-bp non-coding RNAs that fulfill a variety of cellular functions generally by interaction with the 3'-UTR of target genes to suppress their transcription, or translation [5]. Accumulating evidence has demonstrated that miRNAs play crucial roles in EC function and dysfunction, and numerous miRNAs directly or indirectly regulate the expression of a great deal of genes involved in glucose-induced stress [6,7]. The characterization of miRNAs in ECs has opened novel diagnostic and therapeutic options for the prevention of vascular diseases and T2DM. However, it remains a challenge to define the major regulators in these processes. We have previously demonstrated a novel potential biomarker, miR-4463, which showed remarkably elevated expression in the plasma of patients with T2DM and patients with T2DM combined with arteriosclerosis obliterans of low extremity arteries (ASO) [8], suggesting that miR4463 may be involved in the pathogenesis of T2DM and vascular disease. We further discovered that miR-4463 was induced by $\mathrm{H}_{2} \mathrm{O}_{2}$ in a concentration-dependent manner, and miR-4463 promotes $\mathrm{H}_{2} \mathrm{O}_{2}$-induced human umbilical vein EC (HUVEC) apoptosis by enhancing oxidative stress and cellular ROS [9]. However, the function and mechanism of miR-4463 in hyperglycemia has not been investigated to the best of our knowledge, and we sought to explore the role of miR-4463 in diabetes and ischemia.

miRNAs exert functions by interaction with target genes. Among the predicted target genes of miR-4463, protein phosphatase-1 nuclear targeting subunit (PNUTS) attracted our attention. PNUTS, also known as PPP1R10, CAT5, and p99, was originally isolated as a nuclear protein that forms a stable complex with PP1- $\alpha$ and PP1- $\gamma$ in mammalian cells $[10$, 11]. PNUTS plays an essential role in chromatin decondensation, tumor suppression, and DNA damage response by interaction with PP1, retinoblastoma and PTEN [12-14]. It was reported that PNUTS contributes to cellular responses to environmental stress including hypoxia and DNA damage and is a central factor in myocardial ischemia. Forced PNUTS expression protected cardiomyocytes and HUVECs from apoptosis $[15,16]$. These findings suggest that PNUTS may also function in high-glucose- and hypoxia-induced stress, and this needs to be further investigated.

The current study was undertaken to determine the change in miR-4463 expression in patients with combined T2DM and ASO and then to specifically delineate the role of miR4463 in HG and/or hypoxia-induced cell apoptosis in HUVECs. We found that miR-4463 is an upregulated miRNA in the vascular tissues of patients with T2DM and ASO as well as in HUVECs treated with HG and/or hypoxia. We further revealed that downregulation of miR4463 facilitated HUVEC survival under HG and/or hypoxic conditions and elaborated on the probable molecular mechanism. 


\section{Cellular Physiology Cell Physiol Biochem 2018;49:2073-2087 and Biochemistry \begin{tabular}{l|l} 
DOI: 10.1159/000493717 & $\begin{array}{l}\text { (c) } 2018 \text { The Author(s). Published by S. Karger AG, Basel } \\
\text { www.karger.com/cpb }\end{array}$
\end{tabular} \\ He et al.: miR-4463 Regulates Endothelial Cell Injury by Targeting PNUTS}

\section{Materials and Methods}

\section{Characteristics of participants and sample collection}

In total, 16 patients diagnosed with T2DM combined with ASO whose limbs were necrotic and required amputation, and 10 controls from the Affiliated Hospital of Southwest Medical University were enrolled in this study. The diagnosis of T2DM was made according to fasting glucose level $\geq 7.0 \mathrm{mmol} / \mathrm{L} \mathrm{or}$ 2-h postprandial plasma glucose level $\geq 11.1 \mathrm{mmol} / \mathrm{L}$. Individuals with critical illness within the previous 6 months, such as cancer, renal failure, and cardiovascular diseases, were excluded. Pathological femoral arterial tissues were collected after amputation from the 16 patients with ASO and 10 normal femoral arterial tissues were obtained from healthy adults who underwent amputation following an accident. Tissues were immediately preserved in liquid nitrogen until RNA extraction. This study was approved by the Ethic Committee of Southwest Medical University, and informed consent was provided by all patients.

\section{Cell culture}

Primary HUVECs were purchased from ScienCell Research Laboratories (Carlsbad, CA) and grown in complete EC medium (ECM) containing 5\% heat-inactivated fetal bovine serum (FBS), 1\% EC growth factors (ECGS), and 1\% penicillin and streptomycin (P/S). ECM, FBS, ECGS, and P/S were purchased from ScienCell Research Laboratories. Cells at the third to fifth passage in the logarithmic phase were used for all experiments.

T2DM combined with ASO is a complex disease, and in vitro, several stimulating factors such as HG, advanced glycation end products, oxidized LDL, and serum starvation combined with hypoxia have been used to build cell models $[4,17,18]$. In this study, HUVECs were cultured in ECM with 25 mmol/L D-glucose to mimic hyperglycemia (HG group), and in ECM without FBS and ECGS at $1 \% \mathrm{O}_{2}$ to mimic ischemia-induced tissue starvation (Hypoxia group). To simulate hyperglycemia combined with tissue starvation, HUVECs were cultured in HG for $24 \mathrm{~h}$ and then cultured in ECM with $25 \mathrm{mmol} / \mathrm{L} \mathrm{D}$-glucose, $1 \% \mathrm{O}_{2}$, without FBS and ECGS, for $1 \mathrm{~h}, 2 \mathrm{~h}$ and $4 \mathrm{~h}$ (HG + hypoxia group).

\section{Transfection}

miR-4463 mimic (50 nmol/L), miR-4463 inhibitor (100 nmol/L), or its negative control (NC, $100 \mathrm{nmol} / \mathrm{L}$ ) were used in this study. All of the oligonucleotides were purchased from RiboBio Co., Ltd. (Guangzhou, China). After transfection for $12 \mathrm{~h}$, the culture medium was replaced with fresh medium. After $24 \mathrm{~h}$, HUVECs were exposed to HG and/or hypoxia and analyzed using cell viability assay, flow cytometry, Western blot, and immunofluorescence.

PNUTS knockdown was achieved by using PNUTS siRNA according to a previously published study [19]: PNUTS siRNA1, ACAAUUGGCUGACGUAUUC; siRNA2, GCAGACCCGUUCACCAGAA. Our preliminary study showed that siRNA at $100 \mathrm{nmol} / \mathrm{L}$ reduced the PNUTS protein level more effectively than $50 \mathrm{nmol} / \mathrm{L}$; therefore, $100 \mathrm{nmol} / \mathrm{L}$ PNUTS siRNA was used throughout the study. The cells were prepared for further analysis $72 \mathrm{~h}$ after transfection.

\section{RNA extraction and real-time PCR}

Total RNA from plasma and tissues was extracted as previously reported [8]. Input RNA (500 ng) was reverse transcribed to cDNA using a miScript II RT Kit (QIAGEN, Duesseldorf, Germany) according to the manufacturer's instructions. miRNA expression analysis was performed using the miScript SYBR Green PCR kit in an ABI StepOne Plus PCR system (Thermo Fisher Scientific, Waltham, MA, USA). Caenorhabditis elegans miRNA 39 (cel-miR-39) and snRU6 (U6) were selected as reference genes for plasma and tissues respectively. Each experiment was performed in triplicate, and the $2^{-\Delta \Delta C t}$ method was applied to analyze data.

\section{Cell viability assay}

HUVECs were plated at $3 \times 10^{3}$ cells per well in 96-well plates. Cells were transfected and treated as described aforementioned. After that, cells were fixed with $4 \%$ paraformaldehyde for 15 min and stained with DAPI for $10 \mathrm{~min}$. The cell number was calculated using a CellInsight CX5 High Content Screening system (Thermo Fisher Scientific). 


\section{Cellular Physiology Cell Physiol Biochem 2018;49:2073-2087

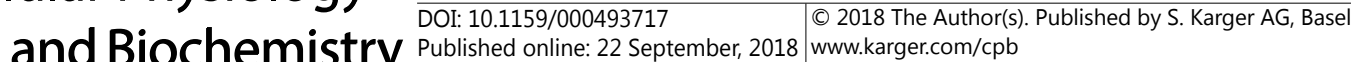 \\ He et al.: miR-4463 Regulates Endothelial Cell Injury by Targeting PNUTS}

\section{Flow cytometry}

Apoptosis assays were performed using an FITC Annexin V Apoptosis Detection Kit (BD Biosciences Pharmingen, San Jose, CA, USA) according to the manufacturer's instructions. Apoptotic cells were analyzed using flow cytometry (BD FACSVerse, BD Biosciences Pharmingen).

\section{Isolation of nuclear extracts}

Nuclear extracts of HUVECs were isolated using a Minute ${ }^{\mathrm{TM}}$ Plasma Membrane Protein Isolation Kit (Invent Biotechnologies, Eden Prairie, MN, USA). Briefly, HUVECs were transfected with NC, miR-4463 inhibitor, or PNUTS siRNA and treated with HG and/or hypoxia. Approximately $2 \times 10^{6}$ cells were collected and resuspended in $200 \mu \mathrm{L}$ buffer $\mathrm{A}$ and then incubated on ice for $10 \mathrm{~min}$. The suspension was transferred to protein extraction filter cartridges after shaking for $30 \mathrm{~s}$. Next, the cartridges were centrifuged at 16,000 $\mathrm{g}$ for $30 \mathrm{~s}$. The pellets were resuspended by vigorously vortexing for $10 \mathrm{~s}$, and then centrifuged at $700 \mathrm{~g}$ for 1 min to obtain the intact nuclei in the pellets. The pellets were lysed in $100 \mu \mathrm{L}$ RIPA lysis buffer and incubated on ice for $30 \mathrm{~min}$ followed by centrifugation at 15, $000 \mathrm{~g}$ for $15 \mathrm{~min}$.

\section{Western blot}

Cells were lysed with RIPA buffer, and $30 \mu \mathrm{g}$ of total protein was loaded and separated on $10 \%$ SDSPAGE gels and transferred onto polyvinylidene difluoride (PVDF) membranes. After blocking with nonfat milk, the membranes were incubated in primary antibody overnight at $4^{\circ} \mathrm{C}$ (anti-PNUTS, $1: 1,000$; anticleaved caspase 3, 1:1, 000; anti-cleaved caspase 9, 1:1,000; anti-PARP, 1:1, 000; anti-Bax, 1:1, 000; antiBcl-2, 1:1, 000; anti-p-AKT [Ser-473], 1:1, 000; anti-AKT, 1:1, 000; anti-p-Bad [Ser-136], 1:800; anti-Bad, 1:800; anti-PTEN, 1:1, 000; all from Cell Signaling Technology, Danvers, MA; or anti-XIAP, 1:3, 000; Abcam, Cambridge, England). GAPDH was used as loading control. Signals were detected after incubation with horseradish peroxidase-conjugated secondary antibodies (1:5, 000, Bioworld, Shanghai, China) for $1 \mathrm{~h}$ at room temperature. Images were scanned using a Fusion solo 4 (Vilber Lourmat Sté, Paris, France).

\section{Immunofluorescence staining}

HUVECs were seeded on 14-mm-diameter poly-L-lysine-coated cover slides, then followed with transfection and hypoxia and/or HG treatment as described above. Then HUVECs were fixed with $4 \%$ paraformaldehyde for $15 \mathrm{~min}$ and treated with $0.2 \%$ Triton X-100 for $8 \mathrm{~min}$. Subsequently, cells were incubated with rabbit anti-PNUTS antibody (1:100) or anti-PTEN antibody (1:100) at $4^{\circ} \mathrm{C}$ overnight. Next, cells were incubated with Cy3 or Alexa Fluor-labeled goat anti-rabbit IgG (1:500) at room temperature for 1 h. Afterwards nuclei were stained with DAPI at room temperature for $10 \mathrm{~min}$. Finally, cells were visualized using a laser scanning confocal microscope (SP8; Leica, Wetzlar, Germany).

\section{Luciferase reporter assay}

A dual luciferase reporter assay was performed in HER 293T cells. Briefly, the human PNUTS wildtype and mutant $3^{\prime}$-UTR were amplified and subcloned into the psiCHECK 2 vector to generate the recombinant plasmid. For the luciferase reporter assay, HER 293T cells were cultured in 96-well plates and co-transfected with the wild type or mutant PNUTS 3'-UTR reporter plasmids and miR-4463 mimic or NC using Lipofectamine ${ }^{\circledR} 2000$ according to the manufacturer's protocol (Thermo Fisher Scientific). After $48 \mathrm{~h}$, the cells were analyzed with a Dual-Luciferase Reporter Assay System (Promega, Madison, WI, USA) according to the manufacturer's instructions with a Lumat LB 9507 luminometer (Berthold, Nashua, USA).

\section{Statistical analysis}

All experiments were performed at least in triplicate. Data are expressed as the mean \pm SD. Demographic and clinical data between the groups were analyzed using Chi-squared and Student $t$ tests. One-way ANOVA followed by the Student-Newman-Keuls test was used to analyze for differences in multiple comparisons. All analyses were performed using SPSS 19.0 software. $P<0.05$ was considered to indicate a statistically significant difference. 


\section{Results}

miR-4463 expression is increased in T2DM combined ASO patients and in HUVECs under $H G$ and/or hypoxic conditions

miR-4463 expression was sharply increased in the vascular tissues of patients with T2DM and ASO (Fig. 1A). To further clarify the effect of hyperglycemia and ischemia on miR-4463 expression, we determined miR-4463 expression in HUVECs after treatment with HG and/or hypoxia. As shown in Fig. 1B and 1C, we found that miR-4463 was dramatically increased in HUVECs exposed to HG or hypoxia. The miR-4463 level markedly increased after treatment with $\mathrm{HG}$ for $24 \mathrm{~h}$ and partially dropped at $48 \mathrm{~h}$ and $72 \mathrm{~h}$, but without a significant difference compared with 24-h treatment. Hypoxia induced elevated miR-4463 at $1 \mathrm{~h}, 2 \mathrm{~h}$ and $4 \mathrm{~h}$. When treated with HG for $24 \mathrm{~h}$ and then hypoxia for $1 \mathrm{~h}, 2 \mathrm{~h}$ and $4 \mathrm{~h}, \mathrm{miR}-4463$ expression sharply increased (Fig. 1D). According to the miR-4463 expression changes, HUVECs were treated in $\mathrm{HG}$ for $24 \mathrm{~h}$ and hypoxia for $4 \mathrm{~h}$ in the following study.

\section{Effect of miR-4463 on HUVEC survival under HG and/or hypoxic conditions}

To investigate the functional role of miR-4463 in HUVECs under HG and/or hypoxic conditions, we first determined the number of HUVECs after transfecting with miR-4463 mimic, inhibitor, or NC followed by treatment with HG and/or hypoxia. We observed that transfection of miR-4463 inhibitor remarkably improved the number of HUVECs in HG and/ or hypoxic conditions compared with transfection of NC, but miR4463 mimic had no obvious effect on HUVEC survival (Fig. 2A). We further evaluated apoptosis in the above conditions, and flow cytometry data indicated that, in $\mathrm{HG}$ and/or hypoxic conditions, downregulation of miR-4463 reduced HUVEC apoptosis compared with the $\mathrm{NC}$ group, while transfection of miR-4463 mimic induced higher levels of apoptosis but without a significant difference compared to the NC group (Fig. 2B). We also determined the expression of apoptosis-related proteins including X-linked inhibitor of apoptosis protein (XIAP), which is a member of the IAP family with the capacity to inhibit apoptosis [20], Bcl-2, Bax, and cleaved caspase 3 that is considered as a critical executioner of apoptosis. As shown in Fig. 3, downregulation of miR-4463 alleviated HG- and/or hypoxiainduced cleaved caspase 3 levels and promoted the expression of XIAP. Furthermore, miR-4463 inhibitor remarkably increased the Bcl-2/Bax ratio. These results indicate that downregulation of

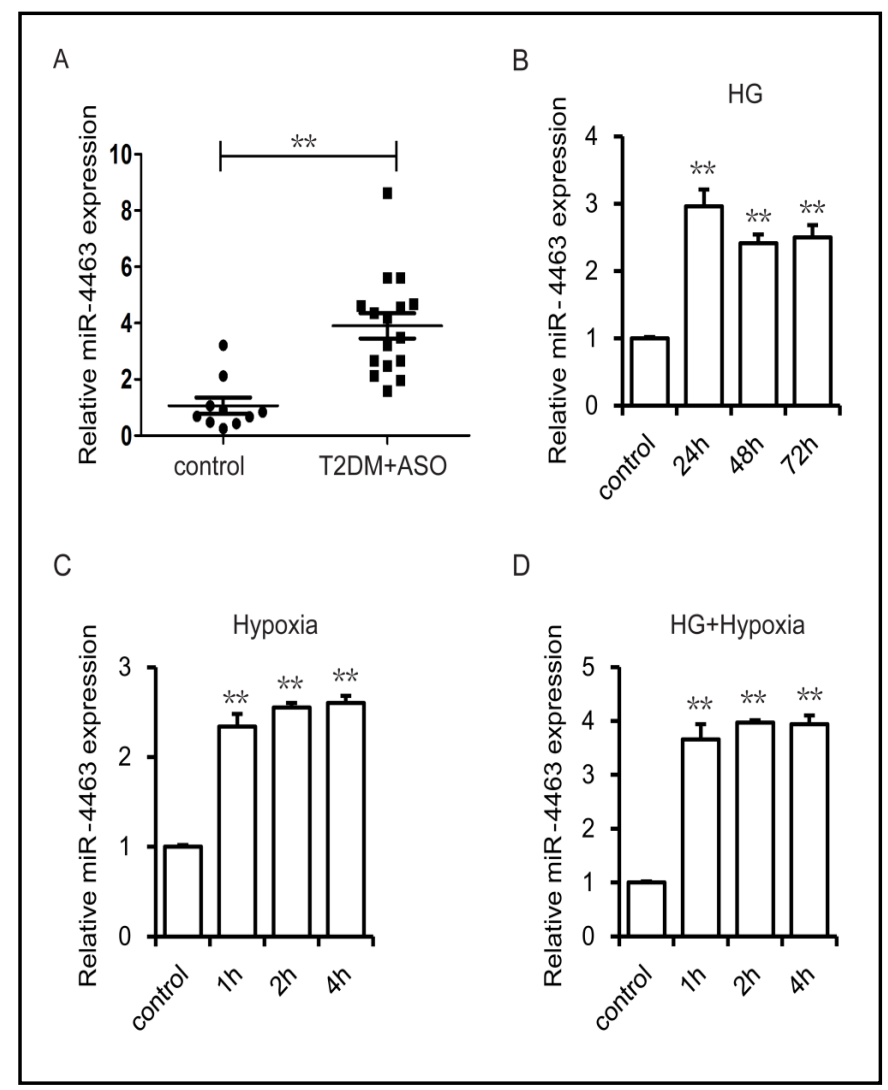

Fig. 1. miR-4463 expression in the vascular tissues of patients with T2DM and ASO (A), and in HUVECs treated with $25 \mathrm{mmol} / \mathrm{L}$ glucose (HG) (B), hypoxia (C), and combined with $\mathrm{HG}$ and hypoxia (D). A, ${ }^{* *} \mathrm{P}<0.01$, compared with each other. $\mathrm{B}, \mathrm{C}, \mathrm{D},{ }^{* *} \mathrm{P}<0.01$, compared with control. 
A

HG

Hypoxia

HG+hypoxia
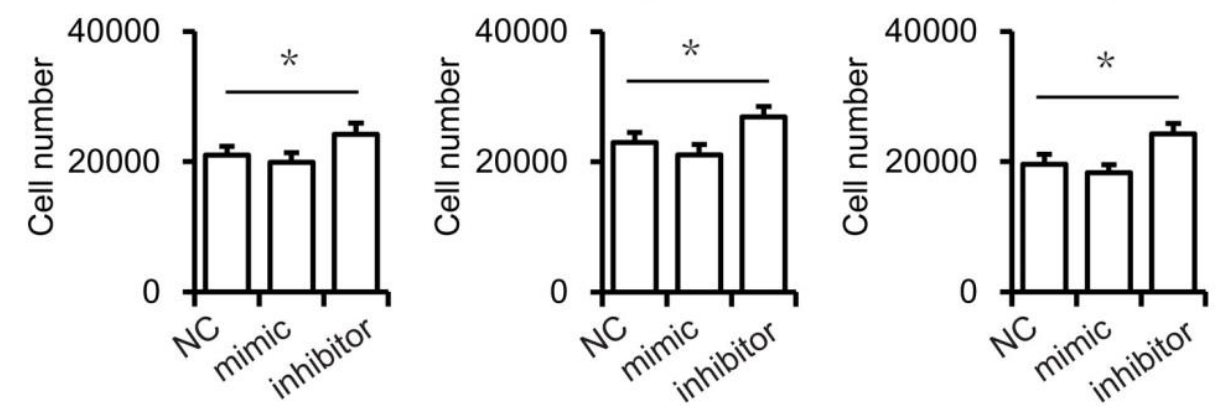

B
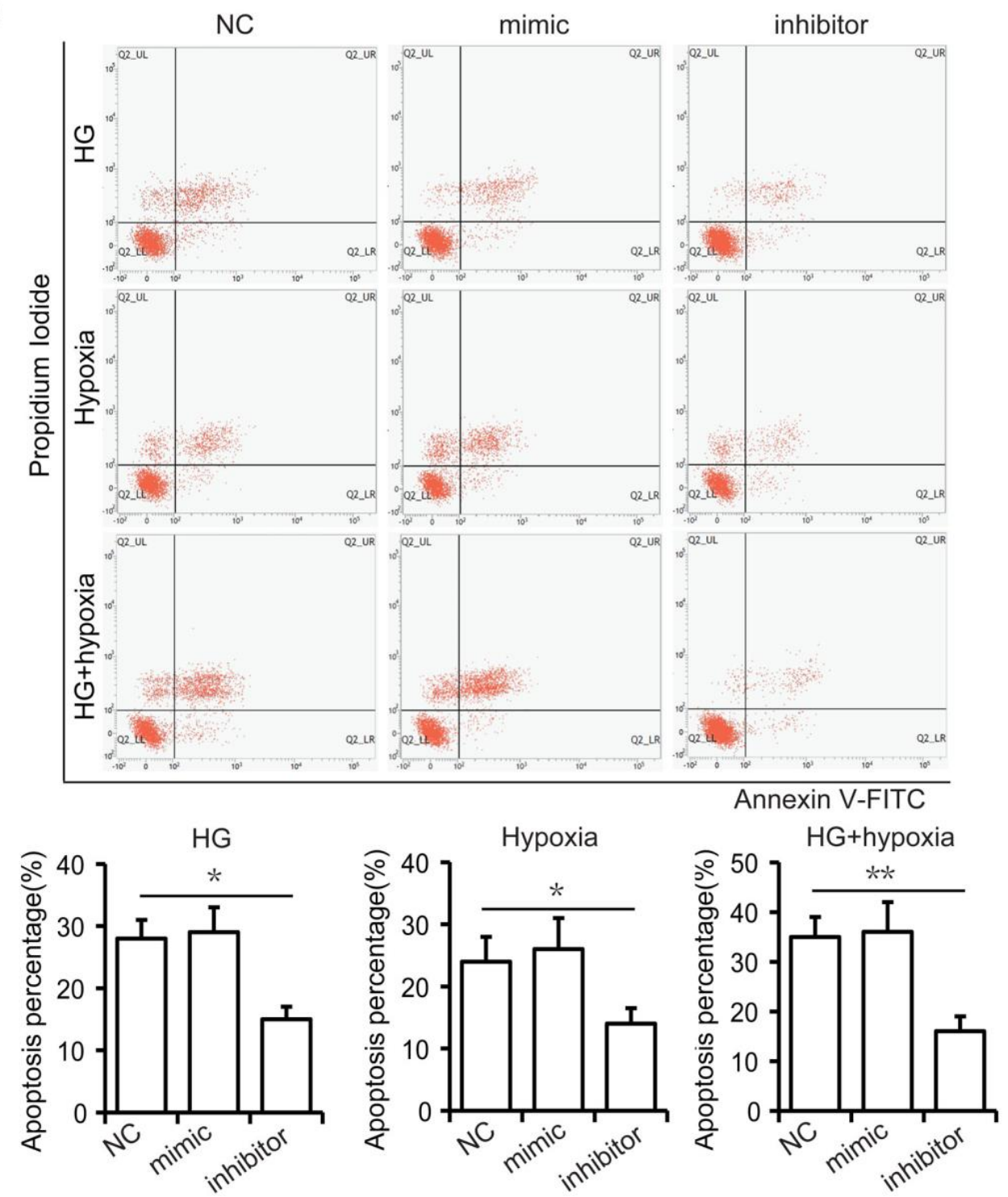

Fig. 2. Downregulation of miR-4463 promotes the HUVEC survival under HG and/or hypoxic conditions. (A) Effect of miR-4463 on HUVEC cell viability under HG and/or hypoxic conditions. ${ }^{*} \mathrm{P}<0.05$, compared with each other. (B) Effect The effect of miR-4463 on HUVEC apoptosis determined using flow cytometry under HG and/or hypoxic conditions. ${ }^{*} \mathrm{P}<0.05,{ }^{* *} \mathrm{P}<0.01$, compared with each other. Mimic, miR-4463 mimic; inhibitor, miR-4463 inhibitor. 
miR-4463 promotes cell survival through decreasing the apoptosis of HUVECs under HG and/or hypoxic conditions.

\section{miR-4463 directly targets PNUTS in HUVECs}

Online miRNA target prediction databases (TargetScan and miRDB) showed that PNUTS was a candidate target gene for miR-4463. The predicted interactions between miR-4463 and its target sites in the PNUTS 3 '-UTR are illustrated in Fig. 4A, which showed good base pairing with the seed region. We next investigated whether miR-4463 could directly bind to the 3'-UTR seed sequence of PNUTS mRNA. We subcloned part of the PNUTS 3'-UTR and its mutated version including the predicted miR-4463 binding sites into a luciferase reporter plasmid. The recombinant plasmid was introduced into HER 293T cells along with miR-4463 mimic or NC. The results showed that overexpression of miR-4463 reduced the

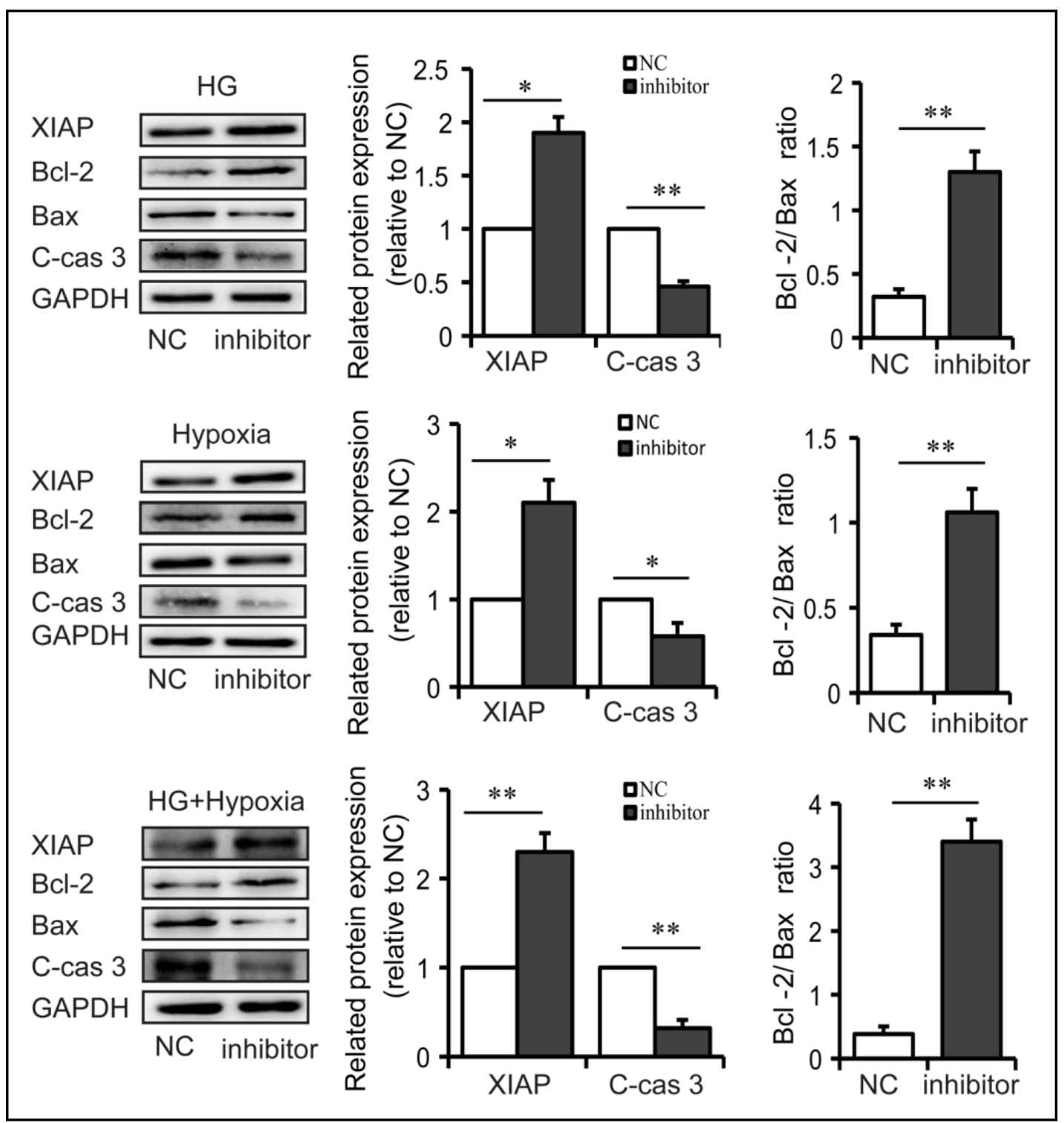

Fig. 3. Expression of apoptosis-related proteins XIAP, Bcl-2, Bax and cleaved caspase 3 (C-cas 3) was determined using Western blot in HUVECs after transfection with NC or miR-4463 inhibitor followed by treatment with $\mathrm{HG}$ and/or hypoxia. ${ }^{*} \mathrm{P}<0.05$, ${ }^{* *} \mathrm{P}<0.01$, compared with each other. 
luciferase reporter activity significantly compared to NC (Fig. 4B). Furthermore, mutations in the PNUTS 3'-UTR that eliminated the predicted binding site with miR-4463 reversed the repression of the reporter activity caused by the overexpression of miR-4463, which suggests that miR-4463 directly targets PNUTS in the $3^{\prime}$-UTR.

Since miR-4463 targeted PNUTS, we next analyzed whether regulation of miR-4463 had any effect on the expression of PNUTS. As shown in Fig. 4C, neither miR-4463 mimic nor inhibitor altered the expression of PNUTS mRNA. However, regulation of miR-4463 altered PNUTS protein expression in HUVECs (Fig. 4D). Taken together, these data suggest that miR4463 directly recognized and targeted PNUTS to negatively regulate its expression at the posttranscriptional level.

\section{Downregulation of miR-4463 promotes HUVEC survival through PNUTS/AKT pathway}

To investigate whether PNUTS is involved in miR-4463-regulated HUVEC apoptosis under HG and/or hypoxic conditions, we examined the expression of PNUTS. As shown in Fig. 5A, PNUTS expression was elevated in miR-4463 inhibitor-transfected HUVECs compared with NC. Recent studies showed that PNUTS could induce AKT phosphorylation and subsequently activate its downstream molecules to influence cell apoptosis [19]. Therefore, we investigated the expression of total AKT, p-AKT, and the downstream total Bad and p-Bad. The results showed that under HG and/or hypoxic conditions the miR-4463 inhibitor induced p-AKT and p-Bad, but without an effect on total AKT and total Bad. In order to elucidate how the enhanced p-AKT level is responsible for the anti-apoptotic effects of downregulation of miR-4463, we inhibited the phosphorylation of AKT with perifosine which could strongly reduce phosphorylation level of AKT. As shown in Fig. 5B, perifosine blocked the miR-4463 inhibitor-induced AKT phosphorylation and increased cleaved caspase 3 expression under HG and/or hypoxic conditions, which indicates that perifosine at least partly blocks the protective role of the miR-4463 inhibitor in HUVEC apoptosis. These

Fig. 4. miR-4463 directly targets PNUTS and negatively regulates its expression. (A) Predicted binding sites of miR4463 within the PNUTS 3'-UTR and its mutated version are shown. (B) Downregulation of luciferase activity by the PNUTS 3 '-UTR is dependent on miR-4463. ${ }^{* *} \mathrm{P}<0.01$, compared with each other. (C) miR4463 has no effect on mRNA expression of PNUTS. (D) miR-4463 negatively regulates expression of PNUTS protein. ${ }^{*} * \mathrm{P}<0.01$, compared with each other.

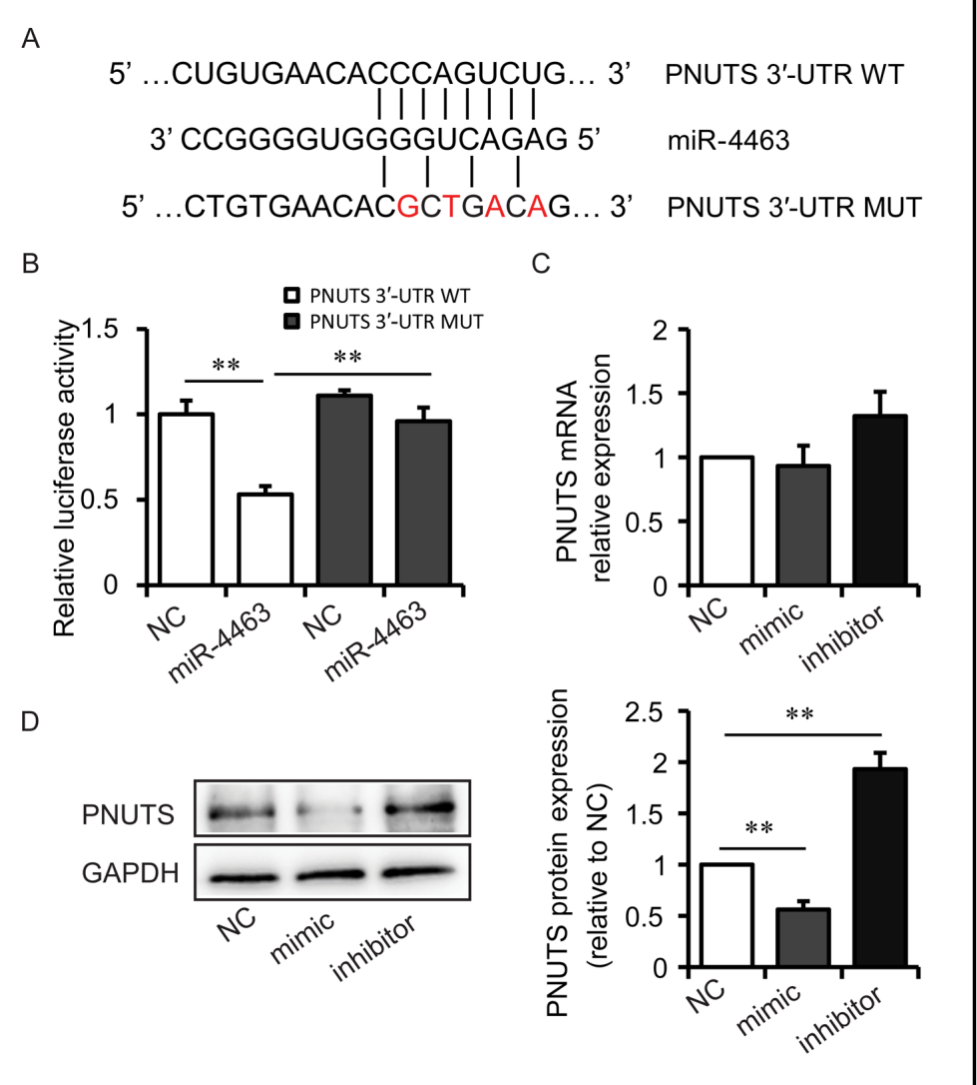




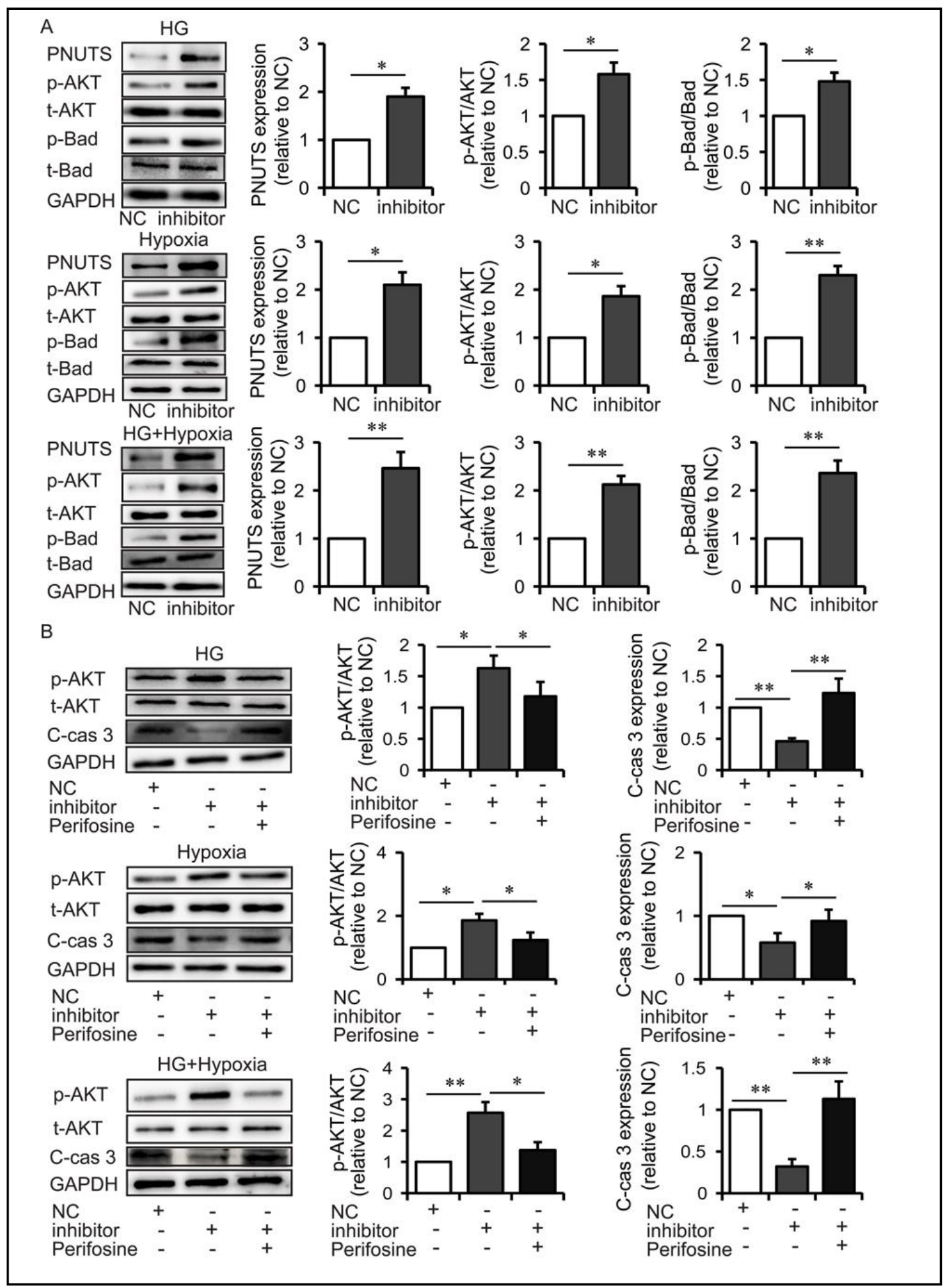

Fig. 5. Downregulation of miR-4463 promotes AKT pathway activation. (A) Expression of total AKT (t-AKT), p-AKT, total Bad (t-Bad), and p-Bad determined using Western blot in HUVECs after transfection with NC or miR-4463 inhibitor followed by treatment with $\mathrm{HG}$ and/or hypoxia. ${ }^{*} \mathrm{P}<0.05,{ }^{* *} \mathrm{P}<0.01$, compared with each other. (B) Inhibition of AKT phosphorylation increases HUVEC apoptosis. Expression of total AKT, p-AKT, and cleaved caspase 3 determined using Western blot in HUVECs after transfection with NC or miR-4463 inhibitor followed by treatment with perifosine under $\mathrm{HG}$ and/or hypoxic conditions. ${ }^{*} \mathrm{P}<0.05,{ }^{* *} \mathrm{P}<0.01$, compared with each other. 


\section{Cellular Physiology Cell Physiol Biochem 2018;49:2073-2087

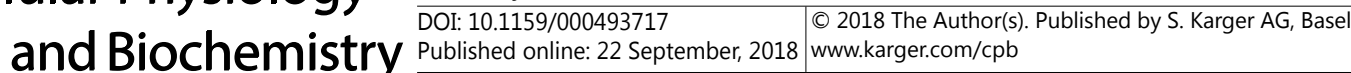 \\ He et al.: miR-4463 Regulates Endothelial Cell Injury by Targeting PNUTS}

results demonstrated that downregulation of miR-4463 protects HUVECs from apoptosis through strengthening AKT signaling pathway.

We further knocked down the expression of PNUTS by siRNA to clarify its role in miR4463-regulated apoptosis in HUVECs. As shown in Fig. 6A, we observed that PNUTS siRNA1 and siRNA2 both robustly inhibited the expression of PNUTS. We chose PNUTS siRNA1 for the following experiments for its better performance. We next detected cell apoptosis when both PNUTS siRNA and miR-4463 inhibitor were transfected in HUVECs undergoing HG and/ or hypoxia treatment and the results showed that silencing of PNUTS had the ability to block the miR-4463 inhibitor-mediated protective effect in HUVECs (Fig. 6B). We further observed that silencing of PNUTS attenuated the phosphorylation of AKT and Bad also facilitating the cleavage of caspase 9 and caspase 3 (Fig. 6C).

Downregulation of miR-4463 promotes PTEN nuclear localization and increased cytoplasmic PNUTS levels

PTEN and PHLPP are phosphatases in AKT pathway. Reduced cytoplasmic and membranal PTEN level decreased the conversion of phosphatidylinositol-3, 4,5-trisphosphate (PIP3), which is required for the activation of AKT, to phosphatidylinositol-4, 5-bisphosphate (PIP2) $[21,22]$. Therefore, cytoplasmic PTEN maintains negative regulation of AKT activation. We next determined the PTEN subcellular localization and cytoplasmic level. As shown in Fig. 7A, transfection of HUVECs with miR-4463 inhibitor promoted PTEN nuclear localization compared with those transfected with NC under HG and/or hypoxic conditions. In addition, greater nuclear localization of PTEN was observed in combined HG and hypoxia stimulation than in cells treated with HG or hypoxia alone. HUVECs co-transfected with PNUTS siRNA1 and miR-4463 inhibitor displayed reduced cytoplasmic PTEN levels compared with those transfected with miR-4463 inhibitor alone, suggesting that silencing of PNUTS inhibits PTEN nuclear translocation. The cytoplasmic PTEN level is negatively associated with the p-AKT level; our previousstudy revealed that the miR-4463 inhibitor promoted AKT phosphorylation, and PNUTS siRNA attenuated this effect. Therefore, we next isolated the cytoplasmic proteins to evaluate whether the miR-4463 inhibitor and PNUTS siRNA influenced the cytoplasmic PTEN level. As shown in Fig. 7B, consistent with immunofluorescence staining, transfection of miR-4463 inhibitor decreased cytoplasmic PTEN levels compared to the NC group while PNUTS siRNA reversed this effect under HG and/or hypoxic condition. Since Kavela S et al. [19] reported that PNUTS specifically binds to PTEN and sequesters PTEN in the nucleus, to clarify the probable function of PNUTS in PTEN translocation in HUVECs, we then investigated PNUTS subcellular localization. As shown in Fig. 7C, PNUTS was almost exclusively located in the nucleus under normal conditions. Under HG and/or hypoxic conditions, we observed that the PNUTS subcellular localization shifted from the nucleus to the cytoplasm in the NC, inhibitor, and inhibitor + siPNUTS groups (et. 7D). Furthermore, compared with the NC group, the miR-4463 inhibitor group showed increased PNUTS cytoplasmic translocation and silencing of PNUTS attenuated this change in subcellular distribution. 


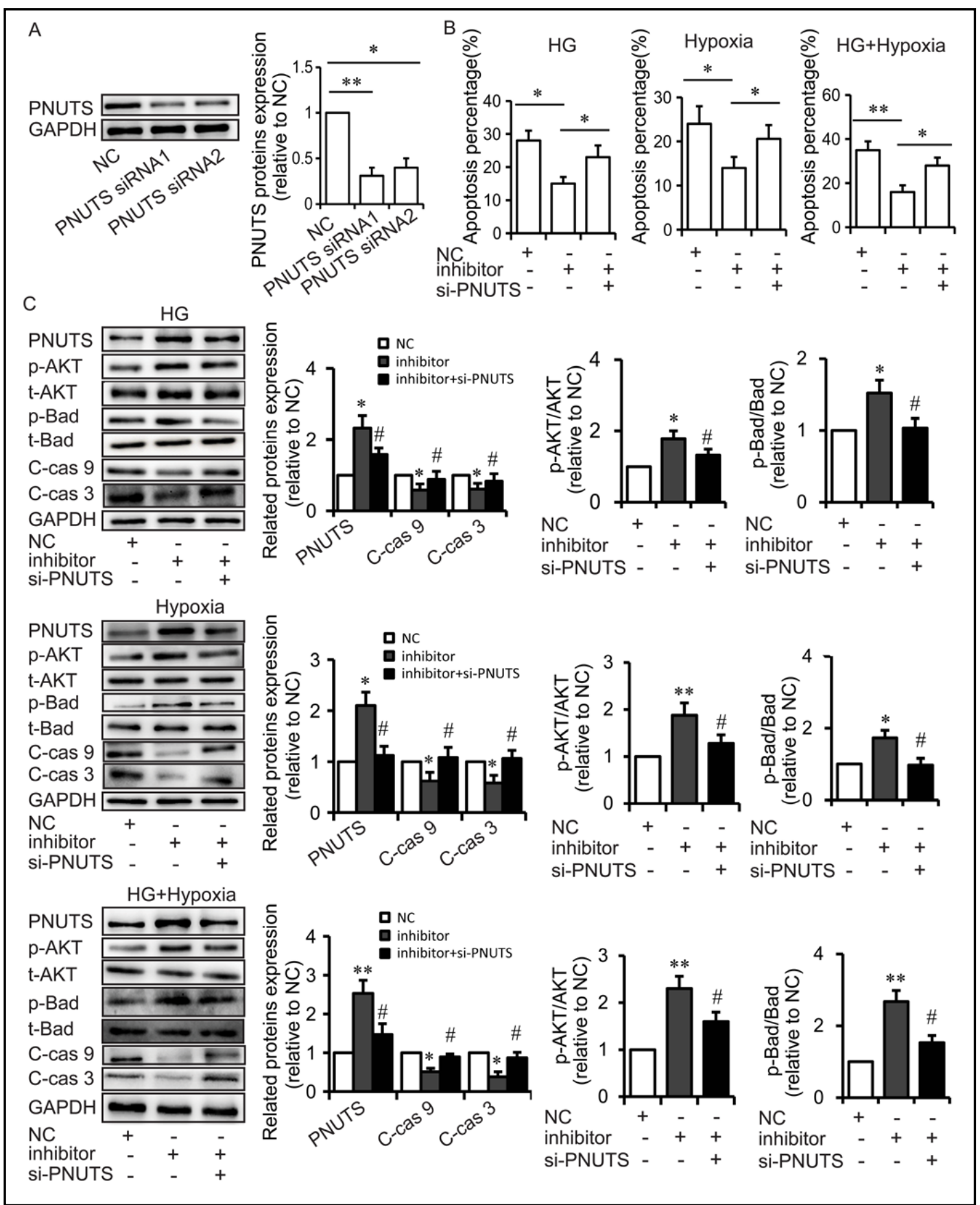

Fig. 6. PNUTS is involved in miR-4463-regulated HUVEC apoptosis in HG and/or hypoxic conditions. (A) PNUTS siRNA decreased the level of PNUTS. ${ }^{*} \mathrm{P}<0.05$, ${ }^{* *} \mathrm{P}<0.01$, compared with each other. (B) HUVEC apoptosis detected by flow cytometry after transfection of NC, miR-4463 inhibitor, or PNUTS siRNA1 followed by treatment with HG and/or hypoxia. ${ }^{*} \mathrm{P}<0.05$, ${ }^{* *} \mathrm{P}<0.01$, compared with each other. (C) Expression of total AKT ( $\mathrm{t}$-AKT), p-AKT, total Bad ( $\mathrm{t}$-Bad), p-Bad, cleaved caspase 9 ( $\mathrm{C}$-cas 9), and cleaved caspase 3 determined using Western blot. ${ }^{*} \mathrm{P}<0.05,{ }^{* *} \mathrm{P}<0.01$, compared with $\mathrm{NC}$. $\mathrm{P}<<0.05$, compared with miR-4463 inhibitor. 


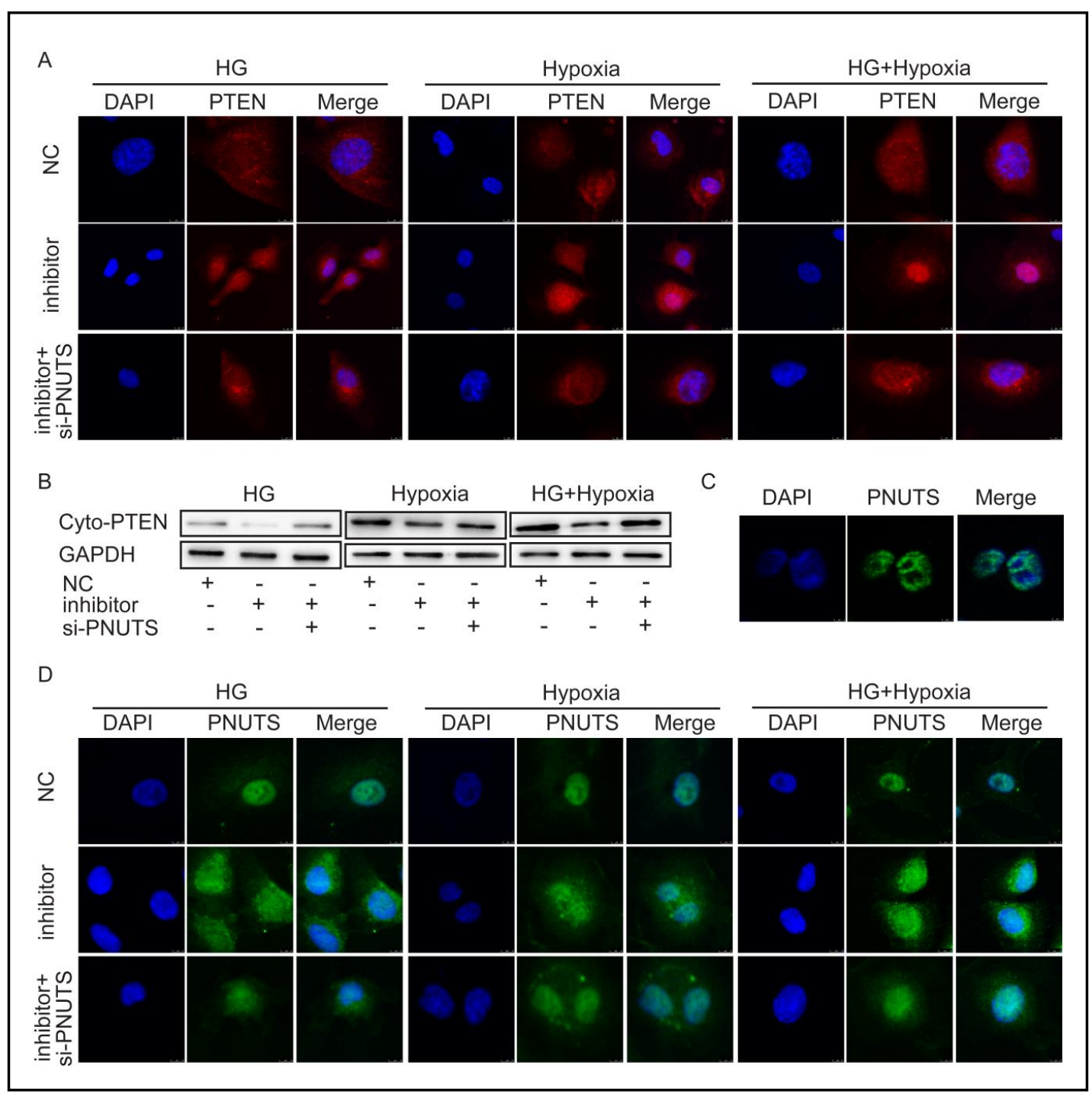

Fig. 7. Downregulation of miR-4463 promotes nuclear PTEN and cytoplasmic PNUTS. (A) Localization of PTEN detected using immunofluorescence staining using anti-PTEN antibody. (B) Cytoplasmic PTEN level detected using Western blot. (C) PNUTS localization in HUVECs under normal culture conditions detected using immunofluorescence staining with anti-PNUTS antibody. (D) PNUTS localization in HUVECs under HG and/or hypoxic conditions detected using immunofluorescence.

\section{Discussion}

Accumulating evidence suggests that EC apoptosis contributes to the onset and progression of diabetes-related vascular complications. The roles of miRNAs as regulatory factors in such processes have drawn increasing attention, but the proposed underlying molecular and cellular mechanisms are still not well understood. Our previous study found that miR-4463 expression was altered in the plasma of ASO patients with or without T2DM. Here, we revealed that miR-4463 was also remarkably upregulated in the vascular tissues of patients with T2DM and ASO as well as in HUVECs treated with HG and/or hypoxia. We provide insight into the role of miR-4463 in HUVEC survival under HG and/or hypoxic conditions, in that downregulation of miR-4463 protected HUVECs from HG- and/or hypoxia-induced apoptosis. Moreover, we have further explored and established the mechanism where miR4463 directly targets PNUTS to regulate the nuclear localization of PTEN, which ultimately

\section{KARGER}




\section{Cellular Physiology Cell Physiol Biochem 2018;49:2073-2087

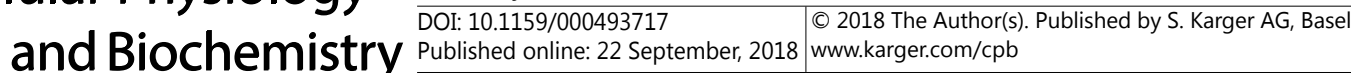 \\ He et al.: miR-4463 Regulates Endothelial Cell Injury by Targeting PNUTS}

impacts AKT signaling pathway to effect HUVEC survival. To our knowledge, this is the first report about the role of miR-4463 in ECs under HG and/or hypoxia.

miRNAs may guide ribosome binding to the $3^{\prime}$-UTR of the target genes and posttranscriptionally inhibit the translation of the target genes. Therefore, the functions of miR-4463 inevitably depend on the target molecules. Here we verified that PNUTS protein level was directly regulated by miR-4463 and it was a direct target of miR-4463. PNUTS has been reported to play an important role in DNA repair and chromosome decondensation [15, 23]. It also has a role in apoptosis in cancer cell lines, ECs and myocardial cells. Knockdown of PNUTS resulted in increased tumor cell apoptosis $[19,24]$ and accumulation of PNUTS enhanced HUVEC and myocardial cell survival under hypoxic conditions [15, 16]. In this study, we confirmed that HG and/or hypoxia induced the expression of miR-4463, and downregulation of miR-4463 protected HUVECs from HG- and/or hypoxia-induced apoptosis by directly targeting and regulating PNUTS and its downstream AKT signaling pathway. Our previous study has revealed that miR-4463 was involved in $\mathrm{H}_{2} \mathrm{O}_{2}$-induced oxidative stress and cell apoptosis in HUVECs and upregulation of miR-4463 exacerbated $\mathrm{H}_{2} \mathrm{O}_{2}$-induced damage [9]. Here, we elaborate on the function of miR-4463 in HUVECs under HG and/or hypoxia, overall, our results at least partly establish the role and the molecular mechanism of miR-4463 in HUVECs which gives us a better understanding of the relationship between altered miR-4463 expression and HUVECs survival under stress.

Previous studies have revealed that PNUTS is a catalytically inactive nuclear-localized protein $[11,19]$. However, Landsverk HB et al. [23] reported that PNUTS was not absolutely localized in the nucleus since it was redistributed throughout the cytoplasm and displaced from chromosomes in prometaphase and metaphase. In our study, we firstly discovered that PNUTS was basically distributed in the nucleus under normal conditions in HUVECs, and it had the capacity to translocate from the nucleus to the cytoplasm under HG- and/or hypoxiainduced stress. Moreover, downregulation of miR-4463 promoted PNUTS translocation and silencing of PNUTS attenuated this effect, indicating that the translocation of PNUTS from the nucleus to the cytoplasm partly depends on the amount of protein. But our data do not address how PNUTS was transported to the cytoplasm, and whether other molecules assisted in this transport process remains unclear. Collectively, our results also support the notion that PNUTS is not exclusively localized to the nucleus but has the ability to translocate from the nucleus to the cytoplasm when necessary, such as when cells are under stress.

Several studies have been reported that PNUTS positively regulates AKT pathway to promote cell growth and proliferation, and suppresses apoptosis in a PTEN-dependent manner. PTEN is an important tumor suppressor primarily localized in the cytoplasm of ECs and may translocate to the nucleus after external stimulation [25]. Cytoplasmic PTEN functions to upregulate the cellular level of PIP3 that is required for the activation of AKT pathway $[21,26]$ that promotes cell growth and survival. Here, we also found that PNUTS positively regulated AKT phosphorylation in a PTEN-dependent manner in HUVECs. Kavela et al. [19] have shown that PNUTS interacts with PTEN specifically in the nucleus. Overexpression of PNUTS enhanced the PTEN nuclear pool, whereas knockdown of PNUTS reduced nuclear PTEN levels by directly interacting with PTEN. Nevertheless, how endonuclear PNUTS induces cytoplasmic PTEN to enter the nucleus remains unclear. In this study, we further elaborated on the regulatory mechanism underlying HG- and/ or hypoxia-stimulated relocalization of PNUTS to the cytoplasm, which was facilitated by downregulation of miR-4463, therefore interacted with and sequestered PTEN in the nucleus to regulate AKT phosphorylation in HUVEC. Silencing of PNUTS attenuated cytoplasmic PNUTS and nuclear PTEN levels, indicating that cytoplasmic PNUTS is essential for PTEN nuclear import. Therefore, we speculate that the mechanism underlying PNUTS regulation of AKT signaling pathway involves PNUTS translocation to the cytoplasm and interaction with PTEN to promote PTEN nuclear transport, which in turn results in increased AKT phosphorylation. However, the mechanism underlying the PNUTS-mediated regulation of PTEN nuclear translocation remains to be further verified. 


\section{Cellular Physiology Cell Physiol Biochem 2018;49:2073-2087

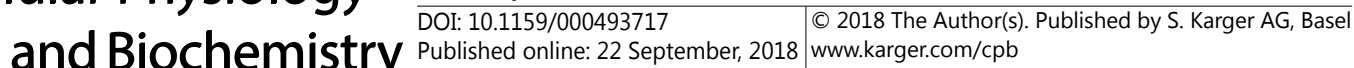

\section{Conclusion}

In conclusion, our data demonstrate that miR-4463 is induced in patients with T2DM as well as in HG and/or hypoxic conditions, and inhibition of miR-4463 reduces HG- and/ or hypoxia-induced EC apoptosis. Moreover, downregulation of miR-4463 enhances PNUTS expression, which promotes PTEN nuclear translocation, with increased AKT phosphorylation and increased cell survival, thus providing a rationale for the anti-apoptotic role of the downregulation of miR-4463. These data reveal that PNUTS is an indispensable downstream effector of miR-4463 and that the miR-4463/PNUTS/AKT axis has profound effects on HG- and/or hypoxia-induced EC apoptosis.

\section{Acknowledgements}

This work was supported by the National Natural Science Foundation of China (Grant number: 81800421), Luzhou Government and Southwest Medical University Project (2017LZXNYD-T10), Collaborative Innovation Center for Prevention and Treatment of Cardiovascular Disease of Sichuan Province, Southwest Medical University, and Support Funds for Training Academic and Technical Leaders in Sichuan (16168).

\section{Disclosure Statement}

The authors declare that no conflict of interests exist.

\section{References}

1 Brownlee M: Biochemistry and molecular cell biology of diabetic complications. Nature 2001;414:813-820.

2 Caporali A, Meloni M, Nailor A, Mitic T, Shantikumar S, Riu F, Sala-Newby GB, Rose L, Besnier M, Katare R, Voellenkle C, Verkade P, Martelli F, Madeddu P, Emanueli C: p75(NTR)-dependent activation of NF-kappaB regulates microRNA-503 transcription and pericyte-endothelial crosstalk in diabetes after limb ischaemia. Nat Commun 2015;6:8024.

3 Hinchliffe RJ, Brownrigg JR, Andros G, Apelqvist J, Boyko EJ, Fitridge R, Mills JL, Reekers J, Shearman CP, Zierler RE, Schaper NC, International Working Group on the Diabetic F: Effectiveness of revascularization of the ulcerated foot in patients with diabetes and peripheral artery disease: a systematic review. Diabetes Metab Res Rev 2016;32 Suppl 1:136-144.

4 Caporali A, Meloni M, Vollenkle C, Bonci D, Sala-Newby GB, Addis R, Spinetti G, Losa S, Masson R, Baker AH, Agami R, le Sage C, Condorelli G, Madeddu P, Martelli F, Emanueli C: Deregulation of microRNA-503 contributes to diabetes mellitus-induced impairment of endothelial function and reparative angiogenesis after limb ischemia. Circulation 2011;123:282-291.

5 Sliwinska A, Kasinska MA, Drzewoski J: MicroRNAs and metabolic disorders - where are we heading? Arch Med Sci 2017;13:885-896.

6 Patella F, Rainaldi G: MicroRNAs mediate metabolic stresses and angiogenesis. Cell Mol Life Sci 2012;69:1049-1065.

7 Silambarasan M, Tan JR, Karolina DS, Armugam A, Kaur C, Jeyaseelan K: MicroRNAs in Hyperglycemia Induced Endothelial Cell Dysfunction. Int J Mol Sci 2016;17:518.

-8 He XM, Zheng YQ Liu SZ, Liu Y, He YZ, Zhou XY: Altered Plasma MicroRNAs as Novel Biomarkers for Arteriosclerosis Obliterans. J Atheroscler Thromb 2016;23:196-206.

-9 Wang X, He X, Deng X, He Y, Zhou X: Roles of miR4463 in H2O2induced oxidative stress in human umbilical vein endothelial cells. Mol Med Rep 2017;16:3242-3252.

-10 Allen PB, Kwon YG, Nairn AC, Greengard P: Isolation and characterization of PNUTS, a putative protein phosphatase 1 nuclear targeting subunit. J Biol Chem 1998;273:4089-4095. 


\section{Cellular Physiology Cell Physiol Biochem 2018;49:2073-2087

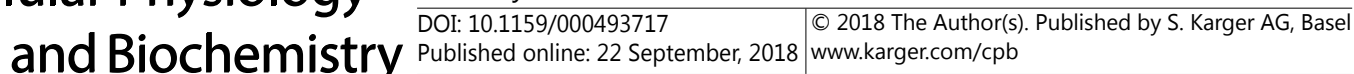

11 Kim YM, Watanabe T, Allen PB, Kim YM, Lee SJ, Greengard P, Nairn AC, Kwon YG: PNUTS, a protein phosphatase 1 (PP1) nuclear targeting subunit. Characterization of its PP1- and RNA-binding domains and regulation by phosphorylation. J Biol Chem 2003;278:13819-13828.

12 De Leon G, Cavino M, D’Angelo M, Krucher NA: PNUTS knockdown potentiates the apoptotic effect of Roscovitine in breast and colon cancer cells. Int J Oncol 2010;36:1269-1275.

13 Grana X: Downregulation of the phosphatase nuclear targeting subunit (PNUTS) triggers pRB dephosphorylation and apoptosis in pRB positive tumor cell lines. Cancer Biol Ther 2008;7:842-844.

14 Verheyen T, Gornemann J, Verbinnen I, Boens S, Beullens M, Van Eynde A, Bollen M: Genome-wide promoter binding profiling of protein phosphatase-1 and its major nuclear targeting subunits. Nucleic Acids Res 2015;43:5771-5784.

15 Lee SJ, Lim CJ, Min JK, Lee JK, Kim YM, Lee JY, Won MH, Kwon YG: Protein phosphatase 1 nuclear targeting subunit is a hypoxia inducible gene: its role in post-translational modification of p53 and MDM2. Cell Death Differ 2007;14:1106-1116.

16 Boon RA, Iekushi K, Lechner S, Seeger T, Fischer A, Heydt S, Kaluza D, Treguer K, Carmona G, Bonauer A, Horrevoets AJ, Didier N, Girmatsion Z, Biliczki P, Ehrlich JR, Katus HA, Muller OJ, Potente M, Zeiher AM, Hermeking H, Dimmeler S: MicroRNA-34a regulates cardiac ageing and function. Nature 2013;495:107110.

17 Cai Y, Liu SX, Xie KL, Zhang WL, Dong L, Liu Y, Zheng Fan, Zhuo C: MicroRNA-492 reverses high glucoseinduced insulin resistance in HUVEC cells through targeting resistin. Mol Cell Biochem 2014;391:117-125.

$>18$ Wang M, Li W, Chang GQ, Ye CS, Ou JS, Li XX, Liu Y, Cheang TY, Huang XL, Wang SM: MicroRNA-21 regulates vascular smooth muscle cell function via targeting tropomyosin 1 in arteriosclerosis obliterans of lower extremities. Arterioscler Thromb Vasc Biol 2011;31:2044-2053.

19 Kavela S, Shinde SR, Ratheesh R, Viswakalyan K, Bashyam MD, Gowrishankar S, Vamsy M, Pattnaik S, Rao S, Sastry RA, Srinivasulu M, Chen J, Maddika S: PNUTS functions as a proto-oncogene by sequestering PTEN. Cancer Res 2013;73:205-214.

20 Qin S, Yang C, Zhang B, Li X, Sun X, Li G, Zhang J, Xiao G, Gao X, Huang G, Wang P, Ren H: XIAP inhibits mature Smac-induced apoptosis by degrading it through ubiquitination in NSCLC. Int J Oncol 2016;49:1289-1296.

-21 Maehama T, Dixon JE: The tumor suppressor, PTEN/MMAC1, dephosphorylates the lipid second messenger, phosphatidylinositol 3, 4,5-trisphosphate. J Biol Chem 1998;273:13375-13378.

22 Reed DE, Shokat KM: INPP4B and PTEN Loss Leads to PI-3, 4-P2 Accumulation and Inhibition of PI3K in TNBC. Mol Cancer Res 2017;15:765-775.

-23 Landsverk HB, Kirkhus M, Bollen M, Kuntziger T, Collas P: PNUTS enhances in vitro chromosome decondensation in a PP1-dependent manner. Biochem J 2005;390:709-717.

24 De Leon G, Sherry TC, Krucher NA: Reduced expression of PNUTS leads to activation of Rb-phosphatase and caspase-mediated apoptosis. Cancer Biol Ther 2008;7:833-841.

-25 Leslie NR, Kriplani N, Hermida MA, Alvarez-Garcia V, Wise HM: The PTEN protein: cellular localization and post-translational regulation. Biochem Soc Trans 2016;44:273-278.

-26 Leslie NR, Batty IH, Maccario H, Davidson L, Downes CP: Understanding PTEN regulation: PIP2, polarity and protein stability. Oncogene 2008;27:5464-5476. 